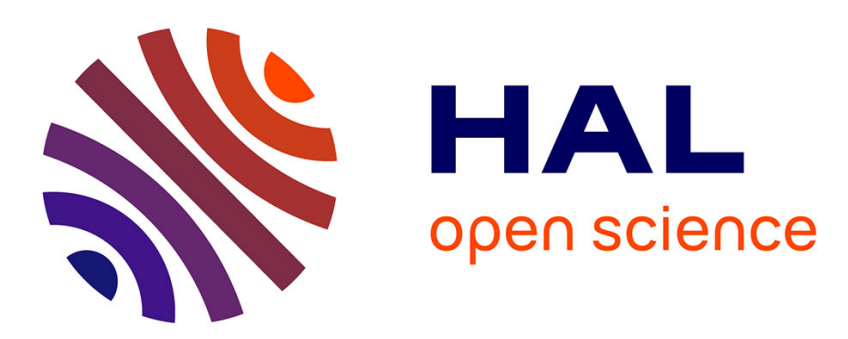

\title{
L'émergence d'un discours de l'innovation managériale? le cas du Balanced Scorecard
}

\author{
Jérôme Méric
}

\section{To cite this version:}

Jérôme Méric. L'émergence d'un discours de l'innovation managériale? le cas du Balanced Scorecard. Comptabilité - Contrôle - Audit, 2003, 9 (3), 10.3917/cca.093.0129 . halshs-01890586

\section{HAL Id: halshs-01890586 \\ https://shs.hal.science/halshs-01890586}

Submitted on 8 Oct 2018

HAL is a multi-disciplinary open access archive for the deposit and dissemination of scientific research documents, whether they are published or not. The documents may come from teaching and research institutions in France or abroad, or from public or private research centers.
L'archive ouverte pluridisciplinaire HAL, est destinée au dépôt et à la diffusion de documents scientifiques de niveau recherche, publiés ou non, émanant des établissements d'enseignement et de recherche français ou étrangers, des laboratoires publics ou privés. 


\title{
L'émergence d'un discours de l'innovation managériale - le cas du Balanced Scorecard
}

\section{Résumé :}

Le balanced scorecard est une technique dont le caractère innovant a été peu discuté, si ce n'est en France. Ce cas particulier suscite donc des interrogations quant à sa nature d'innovation managériale. Pour y répondre, le recours à la terminologie, et plus généralement à des critères ayant trait à la substance de cette technique, se révèle aboutir à une impasse théorique et empirique. Cet article propose une alternative à ces démarches, en se fondant sur l'hypothèse que la nature d'une innovation managériale relève avant tout du discours. L'étude du contexte discursif qui fonde le caractère novateur du balanced scorecard aboutit à la formulation de ce que l'on appelle les « marqueurs » du discours de l'innovation managériale, à savoir la rupture, l'avantage, et la systématicité.

\begin{abstract}
:
The innovating aspect of the balanced scorecard is scarcely discussed, except in France. That is why this particular case arouses many questions as far as its being an innovation is concerned. To answer those questions, terminology and more generally the use of factual and substantial criteria lead to nowhere. This paper aims at proposing an alternative way to define managerial innovations, considering their main nature is discursive. The analysis of the main discourse around the balanced scorecard leads to formulating the three main characteristics of discursive managerial innovations : rupture, advantage, and systematicity.
\end{abstract}

Mots-clés : balanced scorecard, tableau de bord, performance, mesure, innovation

Key words : balanced scorecard, "tableau de bord”, performance, measurement, innovation

Correspondance :

Jérôme Méric

Maître de Conférences

CERMAT - IAE de Tours

Université François Rabelais

50, avenue Jean Portalis

BP 060737206 TOURS Cedex

meric@univ-tours.fr 


\section{Introduction}

Les pratiques managériales des décennies 1980-1990 se sont enrichies d'outils qui, avec un recul très relatif, sont considérées comme des «innovations majeures ». Dans le domaine du contrôle de gestion, il s'agit tout particulièrement des méthodes fondées sur les activités et du balanced scorecard. De ces deux «techniques », celle dont le caractère novateur peut encore susciter le plus de débats est certainement la seconde.

A faire le constat des discussions qu'il provoque, et des attitudes divergentes qu'il suscite dans les mondes professionnel et surtout académique, il semblait intéressant de s'attacher à montrer ce qui, dans le balanced scorecard, constitue éventuellement une « réelle » innovation.

Pour mener à bien une telle démarche, on suppose traditionnellement qu'il existe des «vraies» et des «fausses» innovations managériales. Afin de clairement cerner ces catégories, on utilise alors une définition précise de ce qu'est une innovation managériale. Or les théories et les définitions existantes ne fournissent à cet égard qu'une information partielle. Il faut préciser qu'elles restent très générales non par manque de travaux disponibles ni de réflexion, mais seulement pour préserver la catégorie des innovations, en la débarrassant de toute idée de radicalité.

Face à une démarche qui pourrait être considérée comme une impasse théorique et empirique, nous proposons de concevoir l'innovation managériale, à partir non point de sa substance supposée, mais du discours qui la fonde. Ce discours s'établit alors dans un contexte cognitif particulier, et possède des traits qui le rendent aisément reconnaissable.

La première section de cet article est consacrée à l'étude critique du caractère novateur du balanced scorecard. Elle permet, en analysant cet outil et son émergence, de souligner les limites des approches « en contenu » de l'innovation. Dans la seconde section, une alternative 
à cette démarche est proposée, qui s'appuie sur une typologie des «marqueurs » du discours de l'innovation applicables au balanced scorecard.

\section{L'émergence du balanced scorecard : peut-on définir et identifier une innovation managériale?}

Les sources théoriques sur l'innovation sont nombreuses, mais quasiment aucune ne fait spécifiquement allusion au concept d'innovation managériale. Pourtant cette expression apparaît à de nombreuses reprises, dans des articles, des ouvrages, ou même des titres de thèses. Il semble donc qu'il s'agisse d'une catégorie difficile à définir et à caractériser. Le cas du balanced scorecard, appréhendé au moment de son émergence, permet de souligner les limites d'une telle démarche et l'impasse à laquelle elle peut aboutir. On peut alors lui préférer une approche phénoménologique de l'innovation managériale, fondée sur l'existence d'un discours et d'un contexte favorables à son développement.

\subsection{De la terminologie impossible à l'étude de l'innovation perçue}

La recherche d'une terminologie de l'innovation managériale semble devoir passer par celle de l'innovation en général. Or il semble que ce concept, pourtant très présent dans le discours managérial, demeure extrêmement difficile à cerner et à rendre opérationnel ${ }^{1}$ : une idée, diton, en supposant tout de même qu'elle a lieu de se concrétiser par un renouvellement de la boîte à outils du gestionnaire. Il faut alors s'interroger sur les critères permettant de distinguer une « vraie » innovation managériale d'une « fausse ». Ainsi, pour ne pas restreindre à l'excès le champ de l'innovation, on peut suggérer de distinguer, à l'instar d'Abernathy et Wayne (1974): l'innovation radicale, brutale et induisant des changements importants par l'introduction de nouvelles techniques, voire d'une nouvelle technologie ${ }^{2}$; de l'innovation incrémentale, correspondant aux renouvellements de processus, aux améliorations de gammes de produits, et ainsi de suite. A partir de cette définition élargie, on peut encore s'interroger 
sur ce qui constitue réellement un renouvellement de processus ou de méthodes, mais s'engager dans un tel questionnement n'a pas de fin. Une innovation deviendrait alors assimilable à un changement, rarement radical, qui confronte ou non les individus à un élément inconnu, mais qui dans tous les cas est perçu comme une nouveauté (Durieux, 2001). A cet égard, Godowski (2001) propose une définition élaborée à partir de théories de l'innovation technique :

«Une innovation [managériale] est une idée nouvelle qui peut être soit $1^{\circ}$ ) une recombinaison d'idées anciennes, soit $2^{\circ}$ ) un schéma qui modifie l'ordre du présent, soit $3^{\circ}$ ) une formule ou une approche unique perçue comme nouvelle par les individus concernés. Elle contribue à augmenter le stock de connaissances dont disposent les managers, qui prend la forme d'améliorations ou d'ajouts apportés à l'ensemble des techniques, pratiques et méthodes de gestion, et exerce donc un impact direct sur le mode de management» (Godowski, 2001, p.218).

Dans un contexte socio-économique où l'emploi des termes «innovation » ou «innovant» procède de la pratique incantatoire, on peut être surpris de la modestie de cette définition : une innovation ne serait une innovation que parce qu'elle est perçue comme telle ${ }^{3}$.

C'est donc sur la perception d'un caractère «nouveau » qu'il convient de s'attarder, et non sur les seuls processus (Callon, Latour, 1978). On peut considérer que l'innovation est un changement porteur d'un message « optimiste ». On l'utilise sur le marché comme un vecteur d'image, et l'on ne voit pas pourquoi cela serait fondamentalement différent à l'intérieur des entreprises. L'innovation est caractéristique d'une société qui se veut «en progrès », en puisant sa légitimité dans la technologie et la science. Pourtant, dans le discours qui accompagne la notion de progrès, l'innovation est systématiquement mise en retrait par rapport à l'invention. La première passe traditionnellement pour la recombinaison de techniques existantes, alors que la seconde est censée procéder de l'acte (libre) de création. 
Mais il semble aujourd'hui que cette distinction ne puisse plus avoir cours, à tel point que le discours de l'innovation se substitue à - ou du moins se confond avec - celui de l'invention, et en récupère toute la noblesse symbolique. De fait, l'intellectualisation des pratiques a finalement mis les deux termes sur un pied d'égalité (Sfez, 2002). Le terme d'innovation en général et celui d'innovation managériale en particulier, sont donc censés être porteurs de légitimité, l'objet à légitimer étant un changement de quelque ordre que ce soit.

Quand donc peut-on dire qu'un changement du mode de management est une innovation managériale ? L'étude des théories de l'innovation récentes incite à apporter à cette question une réponse d'ordre phénoménologique : une innovation est un changement qui a été perçu, ou du moins qui est déclaré comme tel. Le minimalisme conceptuel de cette proposition n'autorise pas pour autant l'économie d'une réflexion sur les modalités de cette perception.

La démarche que nous proposons consiste à rapprocher deux objets, l'innovation et le balanced scorecard. En d'autres termes, il ne s'agit pas d'explorer un objet (le balanced scorecard) à partir d'un concept (l'innovation managériale), qui, selon les définitions adoptées, couvrirait une catégorie trop fine (c'est à dire vide) ou trop large. Ce rapprochement doit passer, dans un premier temps, par l'analyse du contexte cognitif et technique de l'émergence du balanced scorecard.

\subsection{Contexte et objet de recherche : l'émergence du balanced scorecard dans la boîte à outils du contrôle}

L'examen du contexte discursif de création du balanced scorecard nécessite un retour sur les quarante dernières années de pratique et de théorie du contrôle de gestion. Cette démarche doit faire face à une difficulté majeure : la spécificité française. De fait, les tableaux de bord, auxquels on rattache habituellement le balanced scorecard, constituent une catégorie d'outils apparemment propre à la pratique française du contrôle de gestion. Plus généralement, on peut 
opposer l'approche «ingénierique» française, à la culture financière nord-américaine (Pesqueux, 2002).

\subsubsection{Les précurseurs du balanced scorecard: indicateurs et tableaux de bord}

Le balanced scorecard (tableau de bord prospectif, dans sa traduction générique), s'inscrit dans la lignée des outils de mesure de la performance. Ses antécédents se fondent sur l'utilisation d'indicateurs plus ou moins structurés, dont l'usage semble s'être développé depuis l'entre-deux-guerres dans de grandes entreprises américaines (Chandler, 1989). L'après-guerre est marquée par un développement de la recherche sur les indicateurs financiers et de gestion ${ }^{4}$. C'est à ce moment qu'est supposée émerger une spécificité française : le tableau de bord. Le développement de cet outil, caractérisé par la recherche d'une structuration des informations, se détache de la recherche internationale, qui se consacre à l'affinement des indicateurs.

\subsubsection{Les indicateurs de gestion}

Les indicateurs de gestion font l'objet d'études qui, dans un premier temps, cherchent à les affiner (Tucker, 1960), et à les articuler avec les programmes d'évaluation des responsables d'entités (Kindall et Gatza, 1963). Dès cette période, on prend en conscience du fait que l'utilisation de telles mesures comporte le risque de mal appréhender les situations d'entreprise et en particulier la productivité (Jasinsky, 1956). Le perfectionnement des indicateurs de gestion a consisté dans un premier temps à développer et affiner l'information comptable, dans un processus qui aboutit à la mise en place d'un système de responsibility accounting $^{5}$. Un second axe de recherche s'attache à compléter la dimension financière par une information de gestion plus proche de la production (i.e. le développement de mesures physiques $^{6}$ ), à la formaliser en recourant aux graphiques (Jason, 1980) et à déterminer des critères «sur mesure » (Rockart, 1979). Pour la productivité, par exemple, des chercheurs parviennent à isoler des facteurs relatifs à l'équipement, à la qualité, à la gestion des stocks, à 
la main d'œuvre, et à la complexité des processus (Hayes \& Clark, 1986, Chew, 1988). Un mouvement de critique plus radical prolonge la recherche d'indicateurs physiques. Le «bottom line » pris à lui seul ne peut pas permettre de comprendre la performance d'un centre de profit, du fait qu'il ne prend pas en compte le potentiel effectif de l'entité considérée, ni les caractéristiques des actifs exploités (Dearden, 1987). De surcroît, la périodicité des mesures financières demeure trop étroitement liée au budget, et ne tient pas compte du délai nécessaire pour appréhender l'effet des décisions managériales (Dearden, 1973, Euske, 1984). Ces critiques se prolongent par l'étude de l'impact comportemental des indicateurs, parfois inattendu et préjudiciable à l'entreprise (Berry, 1983).

La série de travaux sur les indicateurs est singulièrement questionnée par les futurs concepteurs du balanced scorecard, Johnson et Kaplan (1987), qui voient dans l'évolution du contrôle de gestion une progressive «perte de pertinence ». La méthode $\mathrm{ABC}$ semble dans un premier temps fournir un axe de renouvellement des indicateurs fondés sur les coûts (Cooper et Kaplan, 1988 et 1991). L'opposition entre indicateurs financiers et opérationnels n'en est pas moins le fait d'un mode de pensée que certains remettent en cause, en soulignant la difficulté à transcrire les données non-financières en unités monétaires et en proposant des modèles originaux de la performance (Mc Nair et al, 1990).

A faire la synthèse du contexte lié aux indicateurs de gestion, on peut établir que l'évolution de ces outils est passée d'une recherche de perfectionnement par l'enrichissement et l'affinement à une période de remise en cause radicale (voir figure 1). Le balanced scorecard va donc se présenter comme l'une des réponses aux préoccupations les plus récentes. 


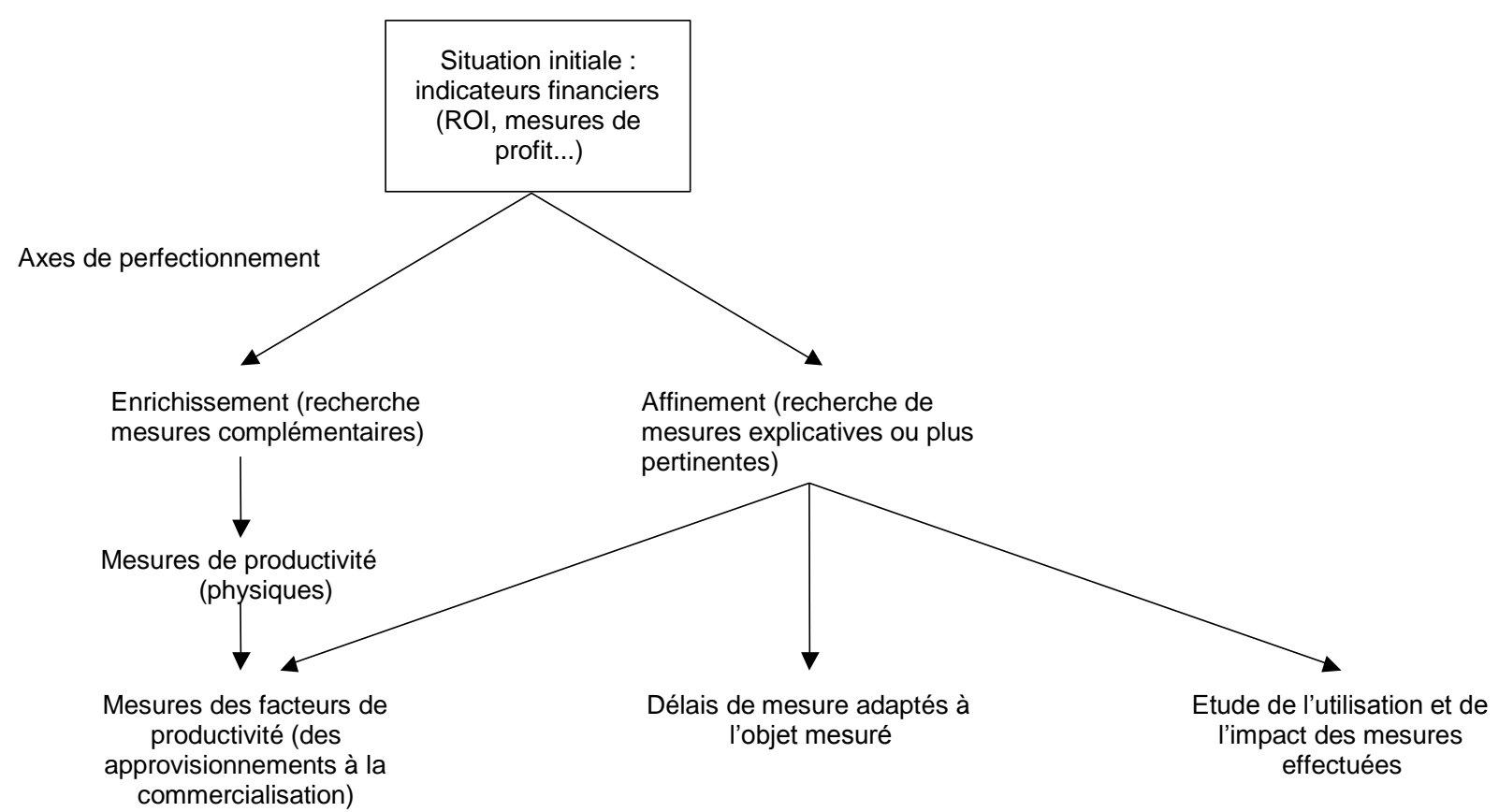

Figure 1 : Axes de développement des indicateurs de gestion (1930-1990)

\subsubsection{Le tableau de bord}

A considérer l'émergence des tableaux de bord dans un contexte français, on constate sa concomitance avec le développement d'une discipline de contrôle de gestion, dans une deuxième moitié des années $1950^{7}$. Les colloques, les articles de recherche et professionnels se multiplient pour aboutir à un premier ouvrage qui met en ordre et consolide l'ensemble de ces acquis. Lauzel et Cibert (1962) définissent le tableau de bord comme « une organisation permanente d'informations destinée à déclencher et à orienter l'action en même temps qu'à illustrer ses effets » (op.cit., p.17) ou encore un outil fournissant «les repères dont la confrontation permet de contrôler l'action passée et oriente vers l'action utile » (op.cit., p.134). Ces « repères » reposent sur une connaissance plus exacte des faits d'ordre interne et externe, le développement de prévisions, et un suivi objectif et précis des réalisations. Le tableau de bord est censé permettre à chaque responsable de cerner sa zone d'intervention, ainsi que les effets de ses actions sur des zones voisines. Encore étroitement lié au budget au moment de sa conception, cet outil se fonde sur des prévisions et des données normatives, afin 
de pointer toute anomalie et tout écart important au moment où ils surviennent. Constitué à partir d'une collecte de mesures présélectionnées, il repose à la fois sur les données internes et les comparaisons interentreprises. Les principes qui président à l'élaboration des tableaux de bord sont la «simplicité » et la «lisibilité » du suivi. Ces deux qualités requièrent une sélection de l'information rigoureuse et adaptée au niveau hiérarchique destinataire.

Dès sa conception, le tableau de bord se distingue donc du simple recueil d'indicateurs par le fait d'être doté d'une structure mettant en rapport l'action et la mesure de ses effets. L'évolution de cet outil demeure étroitement liée à celle des indicateurs. Néanmoins, sa caractéristique est de reposer sur des modèles qui articulent la stratégie d'entreprise et l'action managériale, à quelque niveau hiérarchique que ce soit (de Guerny et al., 1990). Cette approche spécifique permet de distinguer le tableau de bord du reporting traditionnel (Giraud et al., 2002), de même que son caractère sélectif le différencie nettement des systèmes d'information de gestion (Gervais, 2000). La sélection des indicateurs peut reposer sur deux natures de processus distinctes. Soit elle répond immédiatement à une analyse des missions du centre de responsabilité (Gervais, 2000) ou des facteurs-clés de succès pour ce dernier (Echard, 1992), soit elle est déduite de démarches situées en amont de l'élaboration du tableau de bord, et qui se concentrent sur la «modélisation » de la performance. Développée à la fin des années 1980, la méthode OVAR (Objectifs, Variables d'Action, Responsabilités) se fonde sur une déclinaison des objectifs généraux de l'entreprise en plans d'action, ponctuels et réalisables à court terme, lesquels déterminent les indicateurs et leur structuration en tableaux de bord $^{8}$. Les travaux réalisés autour de la mise en œuvre de ces méthodes mettent en évidence l'importance du processus préparatoire à l'élaboration des outils de suivi (Chiapello et Delmond, 1994) et du contexte organisationnel (Busson-Villa, 1996). Au terme de cette démarche, le formalisme à adopter, l'étalonnage des indicateurs alimentent aussi les 
réflexions sur l'utilisation du tableau de bord (Löning et Pesqueux, 1998, Selmer, 1998, Demeestère et al., 2002).

Le tableau de bord a donc vu ses fonctionnalités s'enrichir progressivement, au regard des indicateurs qu'il recèle, et des modèles qui le sous-tendent et le structurent (figure 2). La spécificité du tableau de bord par rapport au reporting ou au simple recueil d'indicateurs contribue à soulever des interrogations quant au caractère novateur du balanced scorecard, et à entretenir le débat à ce sujet.

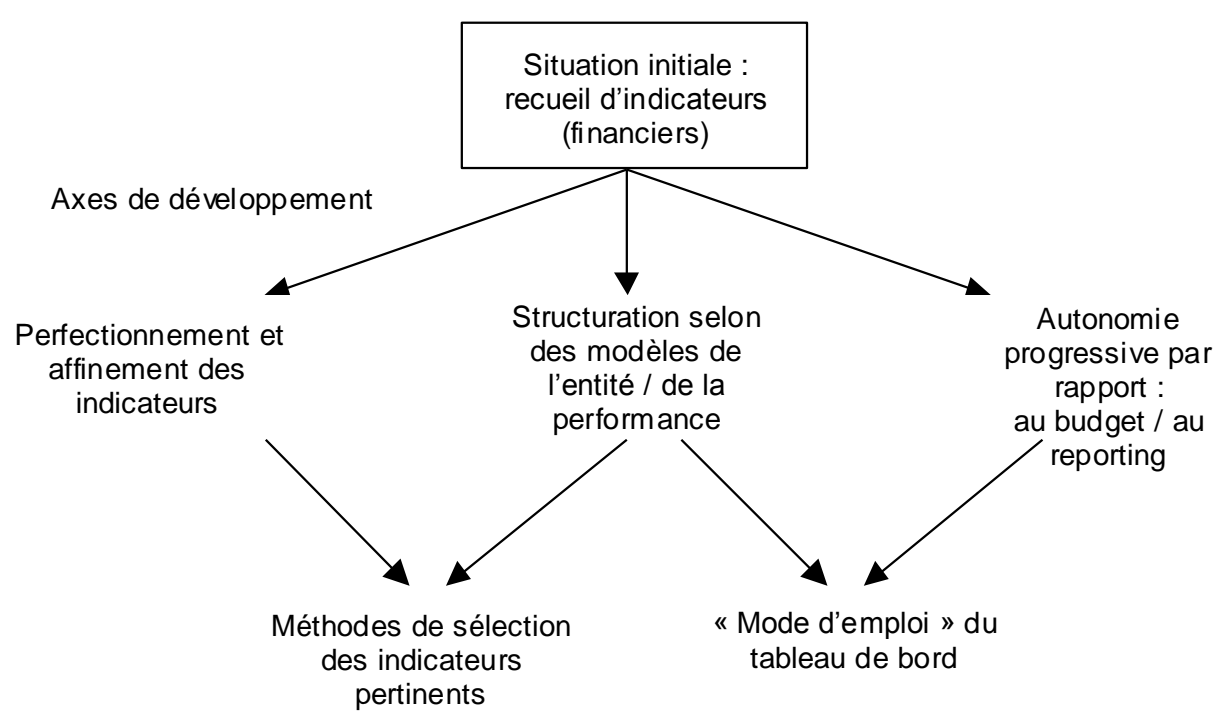

Figure 2 : Axes de développement du tableau de bord (1950-2000)

\subsubsection{Le balanced scorecard: principes et accueil}

Le balanced scorecard est un outil présenté au «public » au début de la décennie 1990. Ses concepteurs en ont depuis détaillé les principes, le contenu, et les méthodes de mise en œuvre. Le succès de cet outil, en particulier depuis la parution du premier ouvrage de synthèse à son sujet (Kaplan et Norton, 1996), est difficilement contestable, même si l'accueil qui lui est réservé par les milieux académiques oscille de l'enthousiasme à la perplexité.

2.2.1. Principes et méthodes du balanced scorecard 
L'article de Kaplan et Norton paru en 1992 se conçoit comme une réponse à la «perte de pertinence $\gg$ du contrôle de gestion signalée dès la fin de la décennie précédente (Johnson et Kaplan, 1987). Cette pertinence perdue tiendrait à la focalisation des mesures de performance sur des aspects financiers. Au débat supposé entre tenants du «tout financier » et du «tout opérationnel », Kaplan et Norton opposent la possibilité de développer un outil qui ne privilégierait aucune dimension au détriment de l'autre. Le balanced scorecard, conçu à partir d'études empiriques (menées entre 1984 et 1992, voir Kaplan, 1994), est présenté comme un «ensemble de mesures qui donnent à la direction une vision rapide mais globale de l'entreprise » (Kaplan et Norton, 1992, p.71). Les mesures financières permettent d'appréhender l'effet des actions déjà entreprises, alors que les critères opérationnels (les axes clients, organisation et apprentissage) permettent d'anticiper ce que sera la performance future de l'entreprise. La nécessaire multiplicité des indicateurs est rapprochée de celle des écrans au tableau de bord d'un avion, dont les informations doivent être en permanence reliées les unes avec les autres. Le balanced scorecard se présente comme un outil de gestion stratégique. Dans un premier temps, il doit permettre d'articuler l'action locale et les buts de l'entreprise. Il se situe ainsi au point de rencontre des processus de contrôle : la déclinaison des objectifs ${ }^{9}$, leur communication, la planification opérationnelle, et le retour d'information (Kaplan et Norton, 1996). Dans une phase de synthèse, le balanced scorecard fournit un cadre pour visualiser la stratégie à l'instar d'une carte : les quatre axes financiers et opérationnels sont présentés comme des territoires sur lesquels des actions sont entreprises, et dont les effets doivent s'enchaîner de manière à accroître la valeur pour l'actionnaire ${ }^{10}$ (Kaplan et Norton, 2000).

Le balanced scorecard est proposé comme un «produit» intégré. Après les principes généraux qui président à sa conception, puis les critères de choix des indicateurs (déclinés selon les quatre axes financiers et opérationnels), la méthode de mise en place de l'outil en 
entreprise est présentée de manière générique, et au travers d'exemples variés ${ }^{11}$. L'objectif de cette méthode est de faciliter la communication, la compréhension, et donc l'utilisation des mesures à quelque échelon que ce soit dans l'organisation (Simons et Davila, 1998).

\subsubsection{Accueil du balanced scorecard.}

Depuis sa formalisation, le balanced scorecard est globalement accueilli comme ce que l'on appelle une « innovation majeure ». Il est ainsi placé au sommet de la hiérarchie des nouveaux outils du manager, comme la comptabilité fondée sur les activités ou la qualité totale. Si l'on étudie encore peu les effets de sa mise en œuvre sur la performance de l'entreprise, il fait l'objet de réflexions sur la manière dont ses principes doivent être enseignés (Bremser et White, 2000). Les critiques de fond qui lui sont adressées ne remettent pas en cause ce caractère novateur (Mooraj et al. 1999, Norreklit, 2000, Jensen, 2002). Plus descriptives, de nombreuses publications relatent la mise en œuvre du balanced scorecard, particulièrement dans le secteur des services, et plus récemment dans l'industrie ${ }^{12}$.

Il est néanmoins intéressant de s'interroger sur l'accueil réservé au balanced scorecard dans un contexte où un outil comparable préexistait ${ }^{13}$. Une première étude transatlantique a présenté les deux outils comme assimilables sous un même vocable (Gray et Pesqueux, 1993). La question est alors de savoir ce que le balanced scorecard apporte en plus. Epstein et Manzoni (1998) précisent que, contrairement aux avis écrits à ce sujet, les critères financiers et l'étalonnage interne dominent encore dans les tableaux de bord français, le principal défaut de ce dernier étant, pour certains, le fait de n'avoir pas su traverser l'Atlantique (Chiapello et Lebas, 1996, Mendoza et Zrihen, 1999, Bontis et al., 1999). Le balanced scorecard ne serait qu'une redécouverte (enrichie) des intérêts du tableau de bord, l'apport essentiel de ce concept tenant à la combinaison des quatre dimensions financières et opérationnelles proposées par Kaplan et Norton (Bouquin, 2001, Demeestère et al., 2002, Pesqueux, 2002). Il est à l'occasion suggéré que la mise en place du balanced scorecard en France obéit à une 
logique de suivisme (Oriot et Misiaszek, 2001). A l'opposé, un courant de pensée défend l'idée qu'il s'agit d'un outil spécifique, distinct des tableaux de bord de gestion traditionnels, et qui s'inscrit dans la lignée plus large des tableaux de bord «stratégiques ». Parmi ces derniers, le balanced scorecard ne serait qu'un modèle accordant encore la primauté aux objectifs financiers $^{14}$ (Selmer, 1998, Gervais, 2000, Wegmann, 2002). Enfin, il est possible de prendre une distance par rapport à ce débat, et de le resituer dans un contexte culturel (Bourguignon et al., 2001), ou encore d'y retrouver les heurs et malheurs de deux métaphores proches et dont les postulats sont discutables (Bessire, 2000)

Si l'examen du discours sur l'outil (et sur ses principes en particulier) ne permet pas d'établir clairement une différence fondamentale entre le balanced scorecard et ce qui l'a historiquement précédé, il est nécessaire de comprendre pourquoi, en tant qu'idée, et en tant que pratique, il est considéré comme une innovation managériale. Comme nous l'avons déjà signalé, les difficultés à établir une terminologie de l'innovation rendent compte des problèmes éprouvés pour opérationnaliser ce concept. C'est au discours que nous attacherons la fonction de déterminer ce qu'est une innovation. Il est donc nécessaire, à partir de l'analyse qui précède, de s'interroger sur ce qui, dans le discours sur le balanced scorecard, en fait une innovation.

\section{Le balanced scorecard et les trois marqueurs du discours de l'innovation : la rupture, l'avantage, et la systématicité}

La perception d'un changement s'effectue dans un contexte donné. Les dimensions contextuelles peuvent être multipliées à différents niveaux, la précision de l'analyse variant selon que l'on s'intéresse à une organisation donnée ou à un phénomène plus général. En considérant le balanced scorecard comme un objet d'étude en lui-même, la première 
dimension à considérer est certainement l'ensemble des connaissances et des techniques de gestion dans lequel cet outil a été introduit. On peut en effet penser que, par contraste avec l'existant, le balanced scorecard s'impose de lui-même comme quelque chose de nouveau. Néanmoins, la situation analysée précédemment incite à explorer une autre hypothèse : celle d'un discours qui détermine la nature d'innovation managériale, et par voie de conséquence, légitime le changement correspondant. C'est ce discours qu'il s'agit à présent de définir et de caractériser.

Nous adopterons l'idée qu'un discours est un «texte contextualisé » (Parret, 1990). S’il ne naît jamais d'un univers discursif vierge, il crée à son tour des contextes discursifs (Jakobson, 1973). Il semble cependant que la notion de contexte soit aussi chargée d'ambiguïtés. Traditionnellement, le contexte est ce qui permet de comprendre ou d'appréhender un dire ou une situation. Ce sont les circonstances (au sens large) de l'acte, langagier ou pas ; plus précisément ce que Goffman (1987) appelle, non sans en critiquer les principes traditionnels, les «présuppositions ». Si, en effet, on cherche dans tout dire un sens caché, un « contexte », des «présuppositions », on s'enferme dans le processus sans fin de recherche de signifiés derrière d'autres signifiés. Afin de lever cette ambiguïté, nous proposons de passer à un autre concept, plus englobant, celui de réalité (non pas celle du pur objet, mais celle que l'on construit) : lorsque nous agissons, nous le faisons par rapport à une réalité, et le discours fait partie intégrante de cette dernière (Austin, 1970).

Il n'est donc nullement question d'aborder la thématique traditionnelle du discours-écran pour laisser planer une suspicion sur le caractère réel d'une innovation managériale, mais bien de considérer le discours comme fondateur de l'innovation (c'est à dire constitutif de sa réalité). L'analyse des contextes cognitifs et techniques de l'émergence et du développement du balanced scorecard a permis d'isoler, dans l'ensemble des discours tenus, les «marqueurs » d'une innovation managériale. Par «marqueurs», nous entendons les traits à la fois 
caractéristiques et constitutifs d'un objet de recherche (Sfez, 2002). Nous avons ainsi identifié trois marqueurs de l'innovation managériale prise en tant que discours : tout d'abord la rupture; puis l'avantage (en regard de l'existant); et enfin la systématicité (l'impératif de généralisation de la pratique). Nous en proposons ici des formulations comme autant d'hypothèses de recherche, et nous les appuyons par la synthèse de propos relevés dans l'analyse contextuelle qui précède.

\subsection{La rupture}

Nous faisons dans un premier temps l'hypothèse que l'innovation managériale repose sur un discours de rupture. Si la terminologie de l'innovation fait souvent le deuil de la radicalité, ce n'est pas le cas du discours qui l'accompagne. C'est d'ailleurs ce qui explique la surprise de ceux qui se penchent pour la première fois sur les définitions attachées aujourd'hui à ce concept, et qui conduit à les qualifier de «minimalistes ». L'innovation se déclare comme une idée, ou encore une technique en rupture avec les idées et les techniques qui l'ont précédée. Le discours de la rupture s'accompagne de traits caractéristiques : le bilan de la pensée et de la pratique du domaine jusqu'à ce jour, une esthétique de la simplicité, et un vocabulaire nouveau.

\subsubsection{Le bilan de la pensée et de la pratique}

« Le bilan de la pensée et de la pratique est à ce jour l'objet de critiques. C'est la nécessité de changer de référentiel qui pousse à la recherche de quelque chose de nouveau ». C'est sur cette hypothèse que semble se fonder le discours de l'innovation. Le bilan en question est dressé dans un contexte qui lui même est présenté comme en rupture avec l'univers dans lequel les pratiques «anciennes » ont été conçues. A ce jour, par exemple, les turbulences de l'environnement et la complexité des stratégies sont censées appeler un renouvellement radical des outils de gestion. 
Le balanced scorecard est ainsi déclaré comme conçu pour les managers désireux de sortir de l'impasse dans laquelle l'utilisation du «tout financier » les a placés. C'est un outil censé être en rupture avec la plupart des systèmes d'information utilisés jusqu'à présent, lesquels ont progressivement perdu toute pertinence. Il se pose aussi comme en rupture avec le débat (i.e. l'impasse) des tenants de l'information financière contre ceux de l'information d'exploitation. Par son approche syncrétique, il suppose que cette discussion n'est pas fondée. Par la mise en relation des dimensions financières, clients, organisationnelles et d'apprentissage, il prétend briser les barrières fonctionnelles. Il constituerait donc un changement fondamental dans les hypothèses sur lesquelles s'appuie la mesure de la performance, qui abandonne le champ du contrôle pour entrer dans celui de la stratégie.

\subsubsection{Une esthétique de la simplicité}

Face à la complication des idées préexistantes, au tumulte du débat, et à la dissonance des pratiques, l'innovation est conçue pour rassembler par la simplicité et l'évidence de l'idée qui la fonde. Cette esthétique, dans sa teneur, est proche de celle du mathématicien cherchant la démonstration la plus économe en enchaînements de formules. Dans un corpus de bonnes pratiques de gestion, on dirait qu'une idée n'est bonne que si elle est simple. Ce principe semble pouvoir être appliqué aux innovations managériales.

Dans le cas du balanced scorecard, la recherche de cette simplicité peut se résumer au travers de quelques questions : pourquoi, par exemple, hésiter entre les approches financières et celles fondées sur l'exploitation, lorsqu'il est tellement plus simple de tenir compte de ces deux dimensions ? Pourquoi multiplier les indicateurs quand une simple trentaine d'entre eux peut entièrement résumer l'activité de l'entreprise, et que l'on peut les regrouper selon quatre dimensions seulement?

\subsubsection{Un vocabulaire nouveau}


La rupture n'est concrètement consommée que lorsque l'innovation s'accompagne d'un vocabulaire distinctif. Ce vocabulaire tient tout d'abord à l'innovation en elle-même. Son nom ne doit pas laisser penser que cet outil a une paternité de quelque ordre que ce soit. Le champ lexical qui l'accompagne doit aussi rendre la rupture plus aisément perceptible. Plus généralement, les termes employés pour décrire l'idée « nouvelle » sont puisés dans le lexique des innovations les plus récentes.

Ainsi, comme l'« inducteur » s'est substitué à l'unité d'œuvre, la « mesure » prend la place de l'indicateur. Le balanced scorecard est un terme apparemment nouveau, qui ne se rattache ni au vocable des indicateurs, ni à celui du dashboard (tableau de bord). A cet égard, la traduction française, qui reprend le terme de tableau de bord, trahit le discours anglo-saxon et pousse à lui trouver un champ d'application spécifique (les tableaux de bord stratégiques, par exemple). Les démarches d'accompagnement de l'outil prennent elles aussi des noms spécifiques, à l'instar de la « cartographie stratégique ». Au delà des termes pris seul à seul, une rhétorique du balanced scorecard semble s'établir (Bourguignon et al., 2000).

L'idée de rupture repose donc sur un ensemble de traits de discours censés suggérer ou démontrer le caractère résolument novateur de l'outil proposé.

\subsection{L'avantage}

L'innovation ne doit pas constituer seulement une rupture par rapport au contexte existant. Encore doit-elle assurer ceux qui l'adoptent de l'avantage qu'elle leur procure, et par là même, les rassurer. Au mieux, cet avantage s'appréhende immédiatement, c'est à dire dès la mise en œuvre de l'idée ; au pire, il vient enrichir le corpus de connaissances du manager (Van de Ven, 1986). D’un point de vue méthodologique, il peut être malaisé de distinguer les composantes «avantage » et « rupture » dans un discours de l'innovation. Il apparaît dans beaucoup des propos étudiés que le changement constitue à lui seul le facteur légitimant: «s'il y a rupture, cela veut dire que c'est innovant, donc nécessairement mieux pour 
l'entreprise ». Ces trois affirmations n'ont besoin que de la première pour s'enchaîner. Néanmoins, au sein même du discours de la rupture, il peut être intéressant de chercher à identifier les variables qui, changeant entre la situation initiale et celle préconisée, sont censées doter le manager de capacités nouvelles. Cet avantage se mesure à l'aune des connaissances d'une part et des pratiques managériales d'autre part.

\subsubsection{L'avantage cognitif}

L'avantage cognitif se définit a minima comme le fait de connaître une approche nouvelle (en rupture avec les précédentes) de l'entreprise. De manière plus spécifique, l'innovation ne peut se déclarer comme telle que parce qu'elle apporte au manager les moyens d'acquérir des connaissances supplémentaires. Ces connaissances doivent pouvoir être utiles à l'avenir. A cet effet, le vocabulaire de l'innovation se fonde sur l'apprentissage, et à ses extrêmes, sur l'épistémologie. Ainsi, le recours au «changement de paradigme» devient assez systématiquement le moyen d'exprimer à la fois la rupture et l'avantage cognitif que cette dernière confère.

En intégrant cette notion dans son modèle de la performance, le balanced scorecard se conçoit comme un outil qui doit sinon favoriser, du moins appréhender l'apprentissage stratégique dans l'organisation. Il est ainsi censé apporter au manager les moyens de s'informer sur lui-même, sur l'organisation et sur son environnement. Il est structuré, nouveau et global ; en cela, il se différencie du simple recueil d'indicateurs qu'est le tableau de bord «à la française », ancien et « local » (Kaplan et Norton, 1996, p. 29). Il se fonde sur un rapport de causalité entre les dimensions financières et opérationnelles, et appréhende ainsi (au moins de manière implicite) la performance de l'entreprise dans son ensemble. Le fait d'identifier les facteurs explicatifs de cette performance permet non plus de la constater ex post, mais de l'anticiper. Le futur devient ainsi un objet de connaissance. L'avantage cognitif tient aussi au 
partage de ces informations, le consensus perceptif devant mener à une meilleure coordination des actions.

\subsubsection{L'avantage pratique}

L'avantage conféré en termes de connaissances ne peut pas à lui seul asseoir la légitimité de l'innovation. Il faut encore que cette dernière crée une avancée significative en termes plus immédiatement pratiques. Son développement est le fruit d'une expérience significative ou d'études empiriques, censées apporter une garantie supplémentaire aux managers. L'évaluation de l'innovation s'arrête avant tout essai d'appréhender l'impact de sa mise en place sur la performance de l'entreprise. Le discours de l'innovation managériale s'arrête souvent aux portes de l'avantage concurrentiel (Porter, 1985), sans vraiment oser les franchir. Le balanced scorecard est décrit comme répondant à ces exigences : parce qu'il apporte des connaissances «nouvelles », et «mieux articulées », il ne peut mener qu'à de meilleures décisions et de meilleures démarches de résolution de problèmes (problem solving). Du fait de sa mise en place, et du partage d'informations qu'il induit, c'est un outil qui permet d'éviter de nombreux conflits, voire d'augmenter l'implication du personnel dans la mise en œuvre de la stratégie. La clarté des mesures prises devrait permettre de faciliter la détermination des responsabilités et des rémunérations variables. En termes plus généraux, l'entreprise gagne en aptitude à formuler et mettre en œuvre sa stratégie.

Dans le discours de l'innovation, l'avantage décrit donc les fonctionnalités cognitives ou pratiques conférées par la mise en œuvre d'une idée nouvelle.

\subsection{La systématicité}

L'innovation tient à la fois sur la rupture et sur l'avantage. Il convient encore de garantir cette rupture et cet avantage des changements de contexte. Quand nous parlons ici de changement, nous nous plaçons dans une optique synchronique: qu'advient-il de l'innovation dans un autre secteur que ceux initialement étudiés ? Que se passe-t-il lorsque l'on essaie de la mettre 
en œuvre dans un autre pays ? Quels effets peut-elle avoir si l'on applique déjà d'autres méthodes novatrices ? Ces questions mettent en avant des facteurs de contingence qui pourraient partiellement remettre en cause l'idée proposée. Le discours de l'innovation oppose à la contingence la systématicité de la technique, dont l'application est donc censée pouvoir se reproduire à l'identique dans des contextes différents. Parmi ces contextes, il convient d'accorder une place particulière aux innovation qui sont déjà présentes «sur le marché ».

\subsubsection{L'adaptation systématique à des contextes divergents}

Afin d'asseoir l'idée d'un modèle parfaitement transférable, un moyen couramment employé est l'exemplarité. A partir du moment où l'on a pu mettre en œuvre l'innovation dans un cadre différent de celui d'origine, la possibilité de l'implanter dans quelque organisation que ce soit est envisageable, si ce n'est assurée. Les seules incompatibilités qui peuvent être mises en évidence concernent des aspects de la pratique de gestion qui doivent être réprouvés.

Le balanced scorecard est présenté comme pouvant échapper à la contingence. Il a été développé dans des grandes entreprises de services et industrielles. Cependant, il est mis en place dans des structures de petite taille ou à but non-lucratif, de même que dans des entreprises dont l'évolution est rapide. Le souci d'articulation de l'action et de la stratégie n'est pas seulement le fait des grandes entreprises. La méthode est aujourd'hui mise en œuvre dans le monde entier, sans changements majeurs de « cartographie ». Il n'est pas incompatible avec les syndicats. En revanche, son application ne serait pas envisageable dans le cadre d'un management autoritaire (Kaplan et Norton, 2001).

\subsubsection{La compatibilité avec d'autres innovations}

D’autres innovations managériales, déjà reconnues et légitimées, pourraient remettre en cause la légitimité de la nouvelle idée proposée. Le discours de l'innovation répond à ce problème en déclinant par le détail les compatibilités, voire les complémentarités qui peuvent exister 
entre ces concepts nouveaux, et surtout leurs applications. Cette démarche présente néanmoins le risque de dévaloriser l'innovation considérée par rapport à celles qui préexistent. Il s’agit alors de rappeler la composante « avantage » déjà analysée.

Le balanced scorecard phagocyte ainsi les «innovations » qui l'ont précédé. La qualité totale et le balanced scorecard sont parfaitement compatibles dès lors que l'on étend les principes de la première dans la dimension «clients » ou «innovation» du second. Cependant, sans balanced scorecard, une démarche de qualité totale n'articule pas suffisamment l'amélioration des processus opérationnels et les résultats positifs pour l'actionnaire. En facilitant la formulation de la stratégie, le balanced scorecard permet de guider les activités de reengineering vers les processus vitaux pour l'entreprise. Il constitue un outil complémentaire à la démarche $\mathrm{ABC}$ (Activity Based Costing), puisque il adjoint aux inducteurs de coûts des inducteurs de performance. Pour sa part, l'EVA (Economic Value Added) founit une mesure pour la dimension financière du balanced scorecard.

Le discours de la systématicité assure à l'innovation la garantie de pouvoir échapper aux contingences. En élargissant le spectre des applications possibles, il complète les dimensions de rupture et d'avantage pour donner l'image d'une solution (à quelques restrictions près) nouvelle, globale, et presque universelle.

\section{Conclusion}

Le développement du balanced scorecard et sa progression vers le sommet des classifications des «meilleures pratiques » constituent des objets d'étude particulièrement éclairants pour qui s'intéresse à l'émergence d'une innovation managériale. Si le modèle (très) lointain de cette dernière est celui de la révolution scientifique, de ce processus qui mène de la main radiographiée de Roentgen à la théorie de la relativité, force est de reconnaître qu'il n'en propose que très rarement la radicalité. Cependant, il est un domaine dans lequel l'innovation 
est plus efficace que sa grande sœur scientifique : il s'agit du délai de sa mise en œuvre. En dépit de termes parfois employés confusément, il faut admettre que le changement de paradigme peut prendre des décennies, alors que l'innovation managériale devient une évidence mondiale en quelques années. Si les hypothèses présentées dans cet article sont valables, cette efficacité tient au vecteur de l'innovation, lequel ne serait ni une pratique ni une théorie, mais un discours, marqué par la rupture, l'avantage, et la systématicité. Ces trois marqueurs légitiment l'innovation. La rupture assure le caractère novateur de l'idée proposée, l'avantage garantit que cette idée ne peut qu'améliorer les pratiques managériales, et la systématicité en prévient toute remise en cause par la contingence ou par d'autres concepts promus au même moment.

Le balanced scorecard se heurte semble-t-il au contexte français. On peut y voir les manifestations d'une « opposition » culturelle et idéologique entre France et Etats-Unis, ou le caractère assez peu novateur de cette technique. Néanmoins, en se demandant s'il s'agit ou non d'une «vraie» innovation, on se soumet au discours de la rupture. Un tel exemple devrait, au contraire, nous inciter à nous demander si l'innovation managériale dispose d'une autre réalité que sa dimension discursive. 
ABERNATHY W.J., WAYNE K. (1974), «Limits of the Learning Curve », Harvard Business Review, septembre-octobre, pp.109-118

AFCOS (Association Française des Conseils en Organisation Scientifique) (1958), Les ratios de gestion, Editions d'Organisation, Paris.

AHN H. (2001), «Applying the Balanced Scorecard Concept : an Experience Report », Long Range Planning, $\mathrm{n}^{\circ} 34, \mathrm{pp} .441-461$.

AUSTIN J.L. (1970), Quand dire c'est faire, traduction et commentaires par G. Lane, Editions du Seuil, Paris.

BERRY M. (1983), Une Technologie invisible? L'Impact des Outils de Gestion sur l'Evolution des Systèmes humains, Cahier de Recherche du Centre de Recherche en Gestion (CRG), Ecole Polytechnique, Paris.

BESSIRE D. (2000), «French Tableau de bord versus American Balanced Scorecard : Misery and Glory of Metaphors », Sixth Interdisciplinary Perspectives on Accounting Conference Proceedings, The University of Manchester, vol 3., ch. 3.14

BONTIS N., DRAGONETTI N.C., JACOBSEN K., ROOS G. (1999), « The Knowledge Toolbox: A Review of the Tools Available to Measure and Manage Intangible Assets », European Management Journal, vol. 17, n4, pp.391-402.

BOUQUIN H. (2001), Le Contrôle de Gestion, PUF, Paris.

BOURGUIGNON A., MALLERET V., NORREKLIT H. (2001), «Balanced Scorecard Versus French Tableau de Bord : Beyond Dispute, a Cultural and Ideological Perspective », Cahiers de recherche du Groupe HEC.

BREMSER W.G. et WHITE L.F. (2000), «An experiential approach to learning about the balanced scorecard », Journal of Accounting Education, ed. 18, pp.241-255.

BROADY-PRESTON J. et HAYWARD T. (1998), « Assessment of the Relationship between Marketing, Information and Strategy Formulation in the UK Retail Banking Sector», International Journal of Information Management, vol.18, Issue 4, pp.277-285.

BUSSON-VILLA F. (1996), «L'utilisation des tableaux de bord dans les PME », Recherches en contrôle de gestion, M. Gervais (Ed.), Economica, Paris, ch7., pp. 213-227.

CALLON M., LATOUR B. (1978), «Comment suivre les innovations ? Clefs pour l'analyse socio-technique », Prospective et Santé Publique, numéro spécial.

CHANDLER A.D. (1989), Stratégies et Structures des Entreprises, Les Editions d'Organisation, Paris.

CHEW W.B. (1988), « No nonsense guide to measuring profitability », Harvard Business Review, janvier-février, pp. 3-9.

CHIAPELLO E. et DELMOND M.H., «Les tableaux de bord de gestion, outils d'introduction du changement », Revue Française de Gestion, janvier-février.

CHIAPELLO E. et LEBAS M. (1996), «The Tableau de Bord, a French Approach To Management Information », European Accounting Association Annual Meeting Proceedings, Bergen, may.

COOPER R. et KAPLAN R.S.(1988), «Measure Costs Right : Make the Right Decisions », Harvard Business Review, septembre-octobre, pp.96-103. 
COOPER R. et KAPLAN R.S.(1991), «Profit Priorities from Activity-Based Costing », Harvard Business Review, mai-juin, pp.2-7.

DEARDEN J. (1973), «Time Span in Management Control », Readings in Cost Accounting, Budgeting, and Control, Fourth Edition, William E. Thomas Ed., South Western Publishing Co, Cincinatti, pp.340-353.

DEARDEN J. (1987), «Measuring Profit Center Managers », Harvard Business Review, septembre-octobre, pp 84-88.

DEMEESTERE R., LORINO P., MOTTIS N. (2002), Contrôle de gestion et pilotage de l'entreprise, Dunod, Paris.

DURIEUX F. (2001), Management de l'innovation, Vuibert, FNEGE, Paris.

EPSTEIN M. et MANZONI J.F. (1998), «Implementing Corporate Strategy : From Tableaux de Bord to Balanced Scorecards », European Management Journal, vol.16, n², avril, pp.190203.

EUSKE K.J. (1984), Management Control : planning, control, measurement, and evaluation, Addison-Wesley.

GERVAIS M. (2000), Contrôle de Gestion, $7^{\text {ème }}$ édition, Economica, Paris.

GIRAUD M., SAULPIC O., NAULLEAU G., DELMOND M.H. et BESCOS P.L. (2002), Contrôle de gestion et pilotage de la performance, Montchrestien, Paris.

GODOWSKI C. (2001), La dynamique d'assimilation des innovations managériales. Le cas des approches par activités dans la banque, Thèse de doctorat es sciences de gestion, Université d'Aix-Marseille, Faculté d'Economie Appliquée.

GOFFMANN E. (1987), Façons de Parler, Traduction par A. Kihm, Editions de Minuit, Paris.

GRAY J. et PESQUEUX Y. (1993), «Comparaison des pratiques récentes de tableaux de bord dans quelques multinationales françaises et nord-américaines », Revue Française de Comptabilité, $\mathrm{n}^{\circ} 242$, pp.61-70.

GUERNY de J., GUIRIEC J.C., LAVERGNE J. (1990), Principes et mise en place du tableau de bord de gestion, $6^{\text {ème }}$ édition, Delmas, Paris.

HAYES R.H., CLARK K.B. (1986), «Why some factories are more productive than others », Harvard Business Review, septembre-octobre, pp.66-74.

JAKOBSON R. (1973), Essais de Linguistique générale, Rapports internes et externes du Langage, Les Editions de Minuit, Paris.

JASINSKY F.I. (1956), «Use and Misuse of Efficiency Controls », Harvard Business Review, juillet-août, pp.105-122.

JASON R.L. (1980), "Graphic Indicators of Operations », Harvard Business Review, novembre-décembre, pp.164-170.

JENSEN M.C. (2002), «Value Maximization, Stakeholder Theory and the Corporate Objective Function », Business Ethics Quarterly, vol.12, n², pp.235-256.

JOHNSON H.T. et KAPLAN R.S. (1987), Relevance Lost: The Rise and Fall of Management Accounting, Harvard Business School Press, Boston.

KAPLAN R.S. (1994), « Management accounting (1984-1994) :development of new practice and theory », Management Accounting Research, vol.5, Issues 3-4, pp.247-260. 
KAPLAN R.S. et NORTON D.P. (1992), «The Balanced Scorecard - Measures that Drive Performance », Harvard Business Review, janvier-février, pp.71-79.

KAPLAN R.S. et NORTON D.P. (1993), «Putting the Balanced Scorecard System to Work », Harvard Business Review, septembre-octobre, pp.134-147.

KAPLAN R.S. et NORTON D.P. (1996), «Using the Balanced Scorecard as a Strategic Management System », Harvard Business Review, janvier-février, pp.75-85.

KAPLAN R.S. et NORTON D.P. (1996), The Balanced Scorecard : Translating Strategy into Action, Harvard Business School Press, Boston.

KAPLAN R.S. et NORTON D.P. (2000), « Having Trouble with Your Strategy ? Then Map It », Harvard Business Review, septembre-octobre, pp.3-11.

KAPLAN R.S. et NORTON D.P. (2001), The Strategy Focused Organization: How Balanced Scorecard Companies Thrive in the New Business Environment, Harvard Business School Publishing, Boston.

KINDALL A.F., GATZA, J.(1963), «Positive Program for Performance Appraisal », Harvard Business Review, Novembre-Décembre, pp.153-160.

LAUZEL P., CIBERT A. (1962), Des ratios au tableau de bord, Editions de l'Entreprise Moderne, Paris.

LÖNING H., PESQUEUX Y. et coll. (1998), Le contrôle de gestion, Dunod, Paris.

Mc NAIR C.J., LYNCH R.L., CROSS K.F. (1990), «Do Financial and Non-Financial Performance Measures Have to Agree ? », Management Accounting, novembre, pp.28-36.

MALO J.L. (1995), «Les tableaux de bord comme signe d'une gestion et d'une comptabilité à la française », Mélanges en l'honneur du Professeur Claude Pérochon, Foucher, Paris, pp.357-376.

MELIONES J. (2000), «Saving Money, Saving Lives», Harvard Business Review, novembre-décembre, pp.5-11.

MENDOZA C. et ZRIHEN R. (1999), «Le tableau de bord, en VO ou en version américaine ? », Revue Française de Comptabilité, n³09, pp.60-66.

MOORAJ S., OYON D., HOSTETTLER D. (1999), «The Balanced Scorecard : A Necessary Good or an Unnecessary Evil ? », European Management Journal, vol.17, n5, pp.481-491.

NORREKLIT H. (2000), «The Balance on the Balanced Scorecard - A Critical Analysis of Some of its Assumptions », Management Accounting Research, n¹1, pp.65-88.

ORIOT F. et MISIASZEK E. (2001), «Mesurer la performance multidimensionnelle : le choix du Balanced Scorecard chez Matra Marconi Space », Echanges, ${ }^{\circ}{ }^{179}$, juillet.

PARRET H. (1990), «Discours », Dictionnaire des notions philosophiques, PUF, Paris, p. 666.

PESQUEUX Y. (2002), Organisation : modèles et représentations, PUF, Paris.

PORTER M.E. (1985), Competitive Advantage: Creating and Sustaining Superior Performance, Free Press, New York.

ROCKART J.F. (1979), "Chief Executives Define their Own Data Needs », Harvard Business Review, mars-avril, pp.80-87.

ROGERS E., SHOEMAKER F. (1971), Communication of Innovations, The Free Press, New York 
RUCCI A.J., KIRN S.P., QUINN R.T. (1998), «The Employee-Customer Profit Chain at Sears », Harvard Business Review, janvier-février, pp. 83-97.

SCHUMPETER J.A. (1936), The Theory of Economic Developement, an inquiry into profits, capital, credit, interest, and the business cycle, Harvard Univeristy Press, Cambridge.

SCHUMPETER J.A. (1942), Capitalism, Socialism and Democracy, New Edition 2000, Harper and Row, Rochester

SELMER C. (1998), Concevoir le tableau de bord, outil de contrôle, de pilotage, et d'aide à la décision, Dunod, Paris.

SFEZ L. (2002), Technique et idéologie - un enjeu de pouvoir, Seuil, Paris.

SIMONS R. et DAVILA A. (1998), «How High is Your Return on Management?», Harvard Business Review, janvier-février, pp.73-80.

TUCKER (1960), Successful Managerial Control by Ratio Analysis, Mc Graw Hill.

VAN DE VAN A.H. (1986), «Central Problems in the Management of Innovation», Management Science, ${ }^{\circ} 32$, pp.590-607.

WEGMANN G. (2002), "Les tableaux de bord stratégiques - analyse théorique et étude de cas longitudinale », Actes des XVI ${ }^{\text {èmes }}$ journées des IAE, Paris, septembre.

\footnotetext{
${ }^{1}$ D'autant que l'on peut difficilement se fonder sur des approches traditionnelles de l'innovation : l'innovation managériale ne provient ni d'un système économique, ni de processus comparables à ceux décrits par Schumpeter $(1936,1942)$.

${ }^{2}$ L'innovation radicale se conçoit lors du développement d'une industrie et non dans une phase de stabilisation de l'économie et du développement.

${ }^{3}$ On peut noter que Rogers et Shoemaker (1971) ont déjà insisté sur la dimension perceptive de l'innovation.

${ }^{4}$ A ce sujet, on peut se référer notamment à Tucker (1960) et AFCOS (1958), qui synthétisent l'ensemble des recherches effectuées dans la décennie précédente.

${ }^{5}$ Pour trouver une analyse détaillée des similitudes entre responsibility accounting et tableau de bord, on se réfèrera à Bouquin (2001) et Pesqueux (2002).

${ }^{6}$ Les mesures physiques sont réputées plus aptes à expliquer la performance financière de l'entreprise, qui n'en serait que la résultante (Jason, 1980).

${ }^{7}$ La métaphore du tableau de bord en gestion semble néanmoins remonter à 1932 (Malo, 1995).

${ }^{8}$ La méthode OVAR, présentée dans une note pédagogique de M. Fiol et H. Jordan (HEC, 1994), est restituée dans des ouvrages plus immédiatement consultables (Löning et Pesqueux, 1998, Giraud et al., 2002).

${ }^{9}$ Le balanced scorecard se décline en outils locaux qui se focalisent sur les mesures plus opérationnelles, tout en rappelant les objectifs globaux qu'elles sont censées servir, il s'agit des scorecards personnalisés (Kaplan et Norton, 1996).

${ }^{10}$ On pourra noter l'application de cette cartographie dans le cadre des activités de service (Rucci et al. 1998).

${ }^{11}$ Du cas Rockwater (Kaplan et Norton, 1993) à une généralisation de la méthode (Kaplan et Norton, 2001).

${ }^{12}$ Pour suivre des applications dans le secteur des services, on peut se référer à Meliones (2000, application dans les services hospitaliers), Broady-Preston et Hayward (1998, dans la banque de détail). Pour l'industrie, on peut consulter, entre autres, les cas de Tetra Pak (Mooraj et al., 1999), d'ABB Industrie (Ahn, 2001)

${ }^{13}$ La traduction « officielle » de balanced scorecard est « tableau de bord prospectif ».

${ }^{14} \mathrm{C}$ 'est à dire des outils spécifiques au contrôle stratégique.
} 